\title{
Changes in transcriptional orientation are associated with increases in evolutionary rates of enterobacterial genes
}

\author{
Chieh-Hua Lin ${ }^{1,2}$, Chun-Yi Lian', Chao Agnes Hsiung ${ }^{1,2^{*}}$, Feng-Chi Chen ${ }^{1 *}$
}

From Ninth Annual Research in Computational Molecular Biology (RECOMB) Satellite Workshop on Comparative Genomics

Galway, Ireland. 8-10 October 2011

\begin{abstract}
Background: Changes in transcriptional orientation ("CTOs") occur frequently in prokaryotic genomes. Such changes usually result from genomic inversions, which may cause a conflict between the directions of replication and transcription and an increase in mutation rate. However, CTOs do not always lead to the replicationtranscription confrontation. Furthermore, CTOs may cause deleterious disruptions of operon structure and/or gene regulations. The currently existing CTOs may indicate relaxation of selection pressure. Therefore, it is of interest to investigate whether CTOs have an independent effect on the evolutionary rates of the affected genes, and whether these genes are subject to any type of selection pressure in prokaryotes.
\end{abstract}

Methods: Three closely related enterbacteria, Escherichia coli, Klebsiella pneumoniae and Salmonella enterica serovar Typhimurium, were selected for comparisons of synonymous $(d S)$ and nonsynonymous $(d N)$ substitution rate between the genes that have experienced changes in transcriptional orientation (changed-orientation genes, "COGs") and those that do not (same-orientation genes, "SOGs"). The $d N / d S$ ratio was also derived to evaluate the selection pressure on the analyzed genes. Confounding factors in the estimation of evolutionary rates, such as gene essentiality, gene expression level, replication-transcription confrontation, and decreased $d S$ at gene terminals were controlled in the COG-SOG comparisons.

Results: We demonstrate that COGs have significantly higher $d N$ and $d S$ than SOGs when a series of confounding factors are controlled. However, the $d N / d S$ ratios are similar between the two gene groups, suggesting that the increase in $d S$ can sufficiently explain the increase in $d N$ in COGs. Therefore, the increases in evolutionary rates in COGs may be mainly mutation-driven.

Conclusions: Here we show that CTOs can increase the evolutionary rates of the affected genes. This effect is independent of the replication-transcription confrontation, which is suggested to be the major cause of inversionassociated evolutionary rate increases. The real cause of such evolutionary rate increases remains unclear but is worth further explorations.

\footnotetext{
* Correspondence: hsiung@nhri.org.tw; fcchen@nhri.org.tw

${ }^{1}$ Division of Biostatistics and Bioinformatics, Institute of Population Health Sciences, National Health Research Institutes, 35 Keyen Road, Zhunan Town, Miaoli County, Taiwan, Republic of China

Full list of author information is available at the end of the article
} 


\section{Introduction}

Genome rearrangements occur frequently in the evolution of prokaryotes. Among the rearrangement events, inversions usually occur symmetrically around the origin (designated as "Ori") or terminus ("Ter") of replication between closely related bacterial genomes [1]. These rearrangement events often lead to changes in transcriptional orientation (designated as "CTOs") [2] and increases in mutation pressure in the affected genes because of a conflict between the directions of transcription and replication $[1,3,4]$. However, when the inversion events result from the flipping across Ori or Ter, replication-transcription conflict may not occur [1]. Furthermore, CTOs may cause changes in homologybased recombination or impediments in DNA replication (by altering, for example, DNA protein binding sites or secondary structure), which may increase mutation rate. Meanwhile, CTOs may lead to disruptions of operon structure and transcriptional regulations, which are potentially deleterious. Therefore, currently existing CTOs may signify relaxation of selection pressure on the affected genes. Nevertheless, it remains unknown whether selection actually plays an important role in maintaining CTOs. In view of the potential influences of CTOs on gene evolution, we are interested in investigating whether CTO per se has any effect on the evolutionary rates of prokaryotic genes.

Note that the evolution of prokaryotic genes is driven by two major forces: mutation and natural selection. An increased mutation rate can accelerate short-term nucleotide substitutions, which are then retained or eliminated by natural selection according to their fitness effects. The evolutionary rates of prokaryotic genes mainly reflect the combinatorial effects of these two forces. In general, substitutions that cause protein sequence changes (nonsynonymous substitutions) are subject to stronger selection pressure than those that do not (synonymous substitutions). By comparing nonsynonymous $(d N)$ and synonymous substitution rate $(d S)$, we may evaluate whether mutation or selection plays a more important role in the evolution of prokaryotic genes.

Here we attempt to examine (1) the effects of CTOs on prokaryotic gene evolution; and (2) the relative contributions of the two abovementioned driving forces to $d S$ and $d N$ in the genes that have experienced CTOs. To this end, we compared the $d S$ and $d N$ between the genes that have experienced CTOs (changed-orientation genes, "COGs") and those that do not (same-orientation genes, "SOGs") in closed related prokaryotic genomes. In the case of mutation-driven evolution, COGs and SOGs should have significantly different $d S$ but approximately the same $d N / d S$ ratios. Alternatively, if selection has been the major driver of the changes in evolutionary rates, the $d N / d S$ ratios are expected to differ significantly between the two gene groups. Clarifying the molecular mechanisms by which prokaryotic genomes evolve may help us understand how prokaryotes develop novel functions, which is in turn relevant to ecological and biomedical studies.

Accordingly, we compared the genomes of three closely related enterobacteria [5,6]: Escherichia coli (ECO), Klebsiella pneumonia (KPN) and Salmonella enterica serovar Typhimurium (STM). A series of analyses were performed to control for potential confounding factors, including gene essentiality, expression level, background mutation rate, the pattern of replication-transcription confrontation, and codon usage bias. These potential confounding factors have been reported to be associated with evolutionary rates. For example, highly expressed genes, essential genes, and genes with large codon usage bias tend to evolve slowly [7-9]. Meanwhile, a higher mutation rate was observed in the genes near $\operatorname{Ter}[10]$ and the genes that were subject to orientation conflicts between DNA replication and transcription [3]. Our results suggest that COGs have significantly higher $d N$ and $d S$ than SOGs. Such increases are independent of the analyzed confounding factors, and are mainly mutation-driven.

\section{Methods}

\section{Data sources}

The complete genomic sequences and gene annotations of Escherichia coli K-12 MG 1655 (GenBank accession number U00096), Klebsiella pneumonia MGH 78578 (CP000647) and Salmonella enterica serovar Typhimurium LT2 (AE006468) were retrieved from the GenBank [11]. These species were selected because their genomes had been completely sequenced and carefully annotated, and because genome-scale gene expression data were available for these species. The orthologous gene pairs were identified according to reciprocal BLASTP best matches with the following parameters: (1) E value $<1.0$ x $10^{-5}$; and (2) >50\% amino acid sequence identity. Only the orthologous genes that were found in all of the three compared species were retained. A total of 2,574 one-to-one orthologous gene groups were therefore obtained for subsequent analyses. Since one of our analyses measured the evolutionary rates separately for the terminal and central regions for individual genes, the analyzed genes must be sufficiently long to reduce variations in evolutionary rate estimates. A previous study suggested that the first 50 and the last 20 codons usually evolved more slowly than the rest $[12,13]$. Therefore, we took twice the length of $70(2 *(50+20)=140)$ to include a central region with a minimal length of 70 codons. 
Accordingly, genes that are shorter than 420 bp (140*3) were discarded. Note that this practice will lead to differences in the number of analyzed gene pairs between different pair-wise species comparisons. The numbers of analyzed gene pairs are listed in Additional file 1. Since the reciprocal-BLASTP approach may not be an optimal solution for finding orthologous genes, we also retrieved orthologous genes for the analyzed species from the OMA database for comparison [14]. In fact, over $98 \%$ of the orthologous gene pairs retrieved from OMA were identical to those identified by using reciprocal BLASTP matches (see Additional file 2). We actually observed similar results when these $98 \%$ of orthologous genes were analyzed (i.e. COGs have significantly higher $d S$ and $d N$ but similar $d N / d S$ when compared with SOGs; all $p<0.05$, see Additional file 3 ).

The locations of Ori and Ter were determined by using the program Oriloc [15].

\section{Estimation of evolutionary rates and statistical analyses}

The amino acid sequences of orthologous gene pairs were aligned by using ClustalW 2.0 [16] and back-translated to nucleotide sequences. $d S$ and $d N$ were estimated by using the CODEML module of PAML 4.1 [17]. Only orthologous gene pairs with a $d S$ value of $<3$ were considered [18]. Note that this will also lead to differences in the number of analyzed genes in different pair-wise species comparisons. In the end, 1,784, 1,809 and 2,069 orthologous gene pairs were obtained, respectively, for the ECO-KPN, STM-KPN, and ECO-STM comparison (Additional file 1). Since $d S>3$ may indicate an extremely high mutation rate and loss of function, we also investigated whether COGs tend to have $d S>3$. Indeed, the proportion of COGs with $d S>3$ is higher than that of all of the analyzed genes (see Additional file 4). This observation is in fact consistent with our results that COGs tend to evolve faster than SOGs.

Spearman's rank correlations and partial correlations between evolutionary rates and the rate-determining factors were performed by using the R program (http:// www.r-project.org). The statistical significance of the evolutionary rate differences was evaluated by using the Wilcoxon Rank Sum test throughout the study.

\section{Analysis of gene expression data}

The genome-scale gene expression data of ECO (GSE15534) and STM (GSE11486) were downloaded from the Gene Expression Omnibus (GEO) database [19]. The gene expression data of KPN were generated by using a custom-made NimbleGen tiling array (provided by Dr. Bernhard O. Palsson at University of California, San Diego). In all of the three data sets, the gene expression levels were measured separately at the log and stationary phase. The signal intensity of gene expression was $\log 2$-transformed and normalized by using quantile normalization. For cross-species comparisons, the gene expression levels for each species were standardized to a median value of 0 and a variance of 1 .

\section{Functional enrichment analysis}

Gene Ontology (GO) enrichment analysis was performed by using the DAVID Bioinformatics Resources $[20,21]$. Since the genes of ECO are more extensively studied than those of the other two species, only the COGs in ECO-STM and ECO-KPN comparisons were included in this analysis. The gene clustering and GO term enrichment were assessed with reference to the enrichment score and the $p$-values of the modified Fisher's Exact test.

\section{Results and discussion}

COGs have higher $d N$ and $d S$ but similar $d N / d S$ ratio when compared with SOGs

In all the pair-wise species comparisons (ECO-KPN, STM-KPN, and ECO-STM), the COGs have significantly higher $d S$ and $d N$ than SOGs (all $p<0.05$ ). However, the $d N / d S$ ratio does not show significant difference between COGs and SOGs in any of the pair-wise comparisons (Figure 1). We also used a three-way (ECOSTM-KPN) comparison to infer lineage-specific COGs, and "completely conserved SOGs" (i.e. genes that never changed transcriptional orientation among the three compared species). As shown in Additional file 5, lineage-specific (ECO-only and STM-only) COGs have significantly higher $d S$ and similar $d N / d S$ ratios when compared with completely conserved SOGs. These observations suggest that the increases in evolutionary rates in COGs are mainly mutation-driven. However, a number of confounding factors need to be excluded to further confirm these results. The confounding factors to be controlled include gene essentiality, gene expression level, background mutation rate, the transcriptionreplication confrontation, and codon usage bias.

\section{Confounding factor 1: gene essentiality}

Essential genes are known to be more evolutionarily conserved than nonessential genes [8]. It is therefore necessary to clarify whether COGs tend to be nonessential, so that they evolve faster than SOGs. We thus retrieved the gene essentiality information of E.coli[22], and divided the orthologous genes into essential and nonessential genes in the ECO-KPN and ECO-STM comparisons. Our results indicate that $d N$ and $d S$ are significantly higher for COGs than for SOGs in nonessential genes (Table 1). We observed similar trends in essential genes, although the differences in $d N$ and $d S$ are statistically insignificant possibly due to the small sample sizes and the reduced variations in evolutionary 


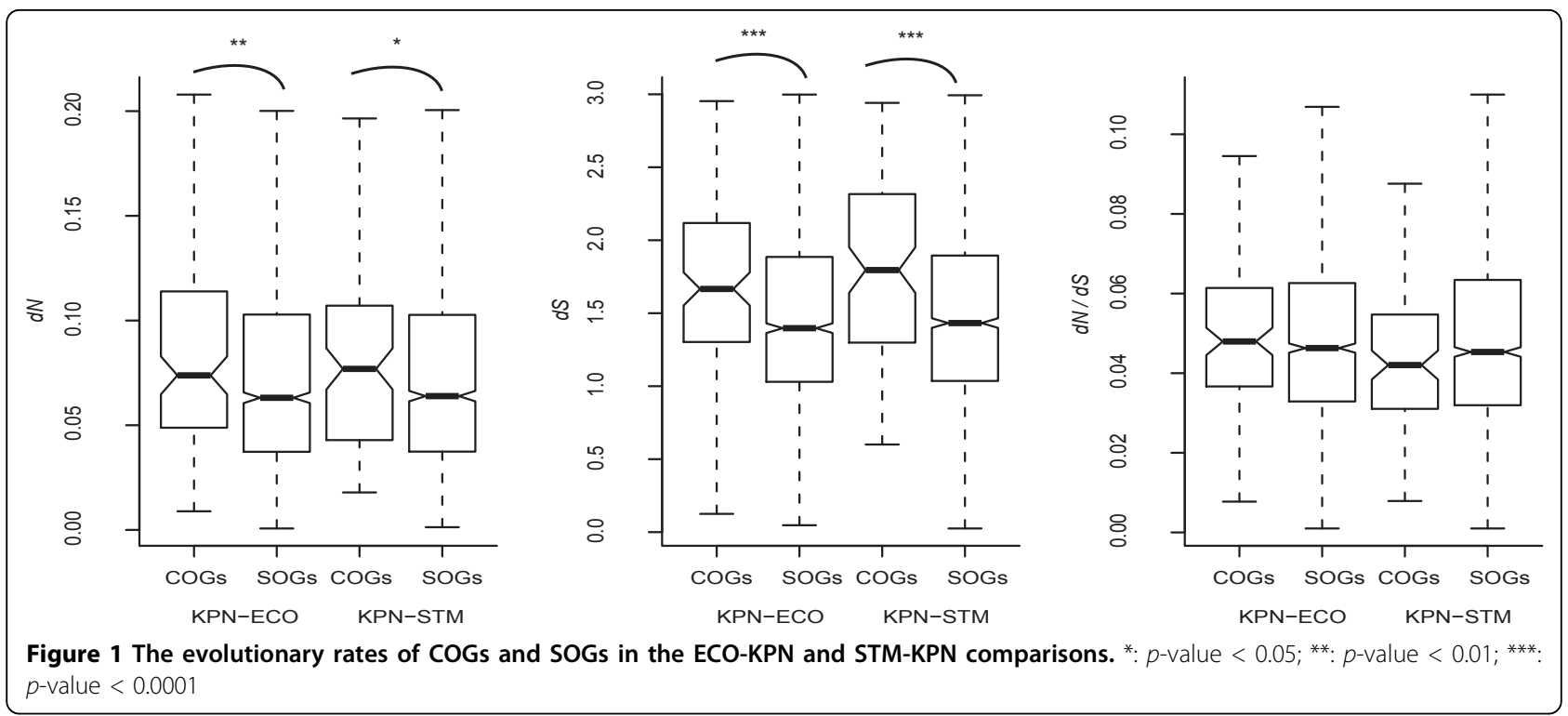

rates in this gene group. Meanwhile, the $d N / d S$ ratios are similar between COGs and SOGs, consistent with our previous observations. Therefore, the difference in the level of gene essentiality does not seem to affect our result. This is expected if the COG-SOG differences in evolutionary rates are mainly mutation-driven.

\section{Confounding factor 2: gene expression level}

Highly expressed genes are under strong selection pressure to maintain functional stability, and thus usually evolve more slowly than lowly expressed genes [23,24]. The question now is whether SOGs tend to be highly expressed, so that they evolve more slowly than COGs. We thus classified the analyzed genes into highly (top $20 \%$ ) and non-highly (other $80 \%$ ) expressed genes. In the ECO-KPN and STM-KPN comparisons, the $d S$ of COGs is significantly larger than SOGs in both highly and non-highly expressed genes for both growth phases (stationary and log phase, Table 1 ). $d N$ values also show similar trends. However, the $d N / d S$ ratios are

Table 1 The median evolutionary rates of COGs and SOGs when different confounding factors are controlled.

\begin{tabular}{|c|c|c|c|c|c|c|c|c|c|c|c|}
\hline \multirow[t]{2}{*}{ confounding factors } & \multirow[t]{2}{*}{ comparison } & \multirow{2}{*}{$\begin{array}{c}\text { \#gene (COG/ } \\
\text { SOG) }\end{array}$} & \multicolumn{3}{|c|}{$d N$} & \multicolumn{3}{|c|}{$d S$} & \multicolumn{3}{|c|}{$d N / d S$} \\
\hline & & & COGs & SOGs & $\begin{array}{c}p^{-} \\
\text {value }\end{array}$ & COGs & SOGs & $\begin{array}{c}p^{-} \\
\text {value }\end{array}$ & COGs & SOGS & $\begin{array}{c}\mathrm{p}^{-} \\
\text {value }\end{array}$ \\
\hline \multicolumn{12}{|l|}{ nonessential gene } \\
\hline & ECO-KPN & $121 / 1426$ & 0.076 & 0.067 & * & 1.6811 & 1.466 & $* * *$ & 0.0481 & 0.0463 & - \\
\hline & ECO-STM & $240 / 1582$ & 0.057 & 0.045 & $* * *$ & 1.5921 & 1.1926 & $* * *$ & 0.0395 & 0.0381 & - \\
\hline \multicolumn{12}{|l|}{ essential gene } \\
\hline & ECO-KPN & $8 / 224$ & 0.045 & 0.041 & - & 1.374 & 0.978 & - & 0.042 & 0.046 & - \\
\hline & ECO-STM & $17 / 225$ & 0.025 & 0.025 & - & 0.785 & 0.825 & - & 0.029 & 0.032 & - \\
\hline \multicolumn{12}{|l|}{$\begin{array}{l}\text { non-highly expressed } \\
\text { gene }\end{array}$} \\
\hline & ECO-KPN (log phase) & $106 / 1240$ & 0.082 & 0.074 & - & 1.721 & 1.561 & $* *$ & 0.049 & 0,047 & - \\
\hline & ECO-KPN (stationary phase) & $103 / 1342$ & 0.077 & 0.066 & - & 1.686 & 1.457 & $* * *$ & 0.048 & 0.046 & - \\
\hline & STM-KPN (log phase) & $88 / 1416$ & 0.077 & 0.068 & - & 1.845 & 1,507 & $* * *$ & 0.042 & 0.045 & - \\
\hline & STM-KPN (stationary phase) & $80 / 1475$ & 0.077 & 0.065 & - & 1.77 & 1.458 & $* * *$ & 0.042 & 0.046 & - \\
\hline \multicolumn{12}{|l|}{ highly expressed } \\
\hline & ECO-KPN (log phase) & $23 / 415$ & 0.045 & 0.037 & - & 1.352 & 0.896 & $* *$ & 0.042 & 0.044 & - \\
\hline & ECO-KPN (stationary phase) & $26 / 313$ & 0.064 & 0.047 & - & 1.459 & 1.142 & $*$ & 0.047 & 0.045 & - \\
\hline & STM-KPN (log phase) & $18 / 287$ & 0.06 & 0.037 & - & 1.384 & 0.895 & $*$ & 0.046 & 0.046 & - \\
\hline & STM-KPN (stationary phase) & $26 / 228$ & 0.075 & 0.053 & * & 1.845 & 1.273 & $* * *$ & 0.042 & 0.044 & - \\
\hline
\end{tabular}


approximately the same between COGs and SOGs (Table 1). Notably, it has been previously reported that mutation rate may increase with gene expression level [25-27]. Therefore, it is of interest to investigate whether the increases in evolutionary rate in COGs are associated with increased expression levels in these genes. However, our results indicate that this is not the case. Highly expressed COGs actually have lower $d N$ and $d S$ than non-highly expressed COGs (Additional file 6). Therefore, the increases in evolutionary rates (particularly $d S$ ) in COGs do not result from increased expression levels in these genes.

\section{Confounding factor 3: background mutation rate}

Although gene inversions usually occur in the vicinity of both Ori and Ter[1,2], we actually found that most COGs analyzed in this study were located near Ter (Figure 2). It is known that the genes located near Ter have an increased mutation rate [28]. We then ask whether the uneven chromosomal distribution of COGs has contributed to the COG-SOG differences in evolutionary rates. Therefore, we performed partial correlation analyses to examine the relationship between evolutionary rates and gene type (COG or SOG). Our results indicate that $d S$ is significantly correlated with the COG/SOG gene type even when the distance effect is controlled (Spearman's $\rho>0.05$ and $p<0.05$ ) (Additional file 7). The positive correlation indicates that CTOs have a location-independent effect on the increase of $d S$. Meanwhile, the distance-controlled correlations are statistically insignificant between $d N$ (and also $d N / d S$ ) and the COG/SOG gene type. Together, these results indicate that CTOs actually have a location-independent effect on $d S$. Meanwhile, the increase in $d N$ in COGs is likely affected by the chromosomal locations of these genes. However, since the COGs that are close to Ter far outnumber those that are not, more evidence is needed to clarify the correlation between $d N$ and the COG/SOG gene type in the context of background mutation rate.

\section{Confounding factor 4: replication-transcription confrontation}

The head-on collision between the directions of transcription and replication has been reported to increase the mutation rate of the affected genes $[3,10,29]$. When a gene is involved in an inversion event, its transcriptional orientation is likely to change, causing replication-transcription confrontation. We thus investigated whether COGs have replication-transcription confrontation more often than SOGs. Interestingly, most of the COGs maintain their status in terms of replication-transcription confrontation. For example, using KPN as an outgroup, we found that 157 of 162 COGs in the ECOSTM comparison maintain the status of head-on collision or co-orientation between replication and transcription after the occurrences of the inversion events. Meanwhile, the ratio of head-on-collision to co-orientation genes in COGs and SOGs are 1.1 and 0.64, respectively in the ECO-STM comparison. Therefore, COGs are in fact more likely to be head-on-collision genes than SOGs. To further clarify whether the difference in the degree of head-on collision is a major determinant of the COG-SOG differences in evolutionary rate, we compared the evolutionary rates of COGs and SOGs by controlling the replication-transcription confrontation

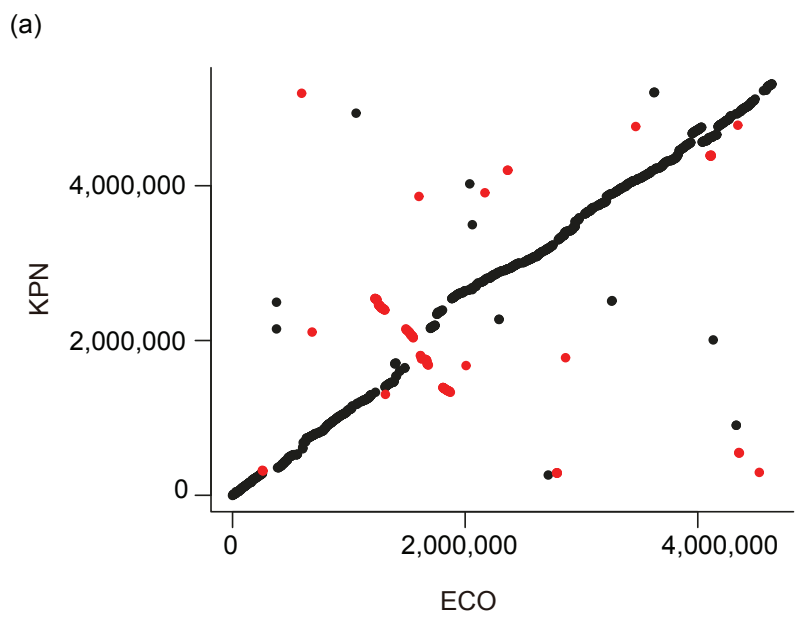

(b)

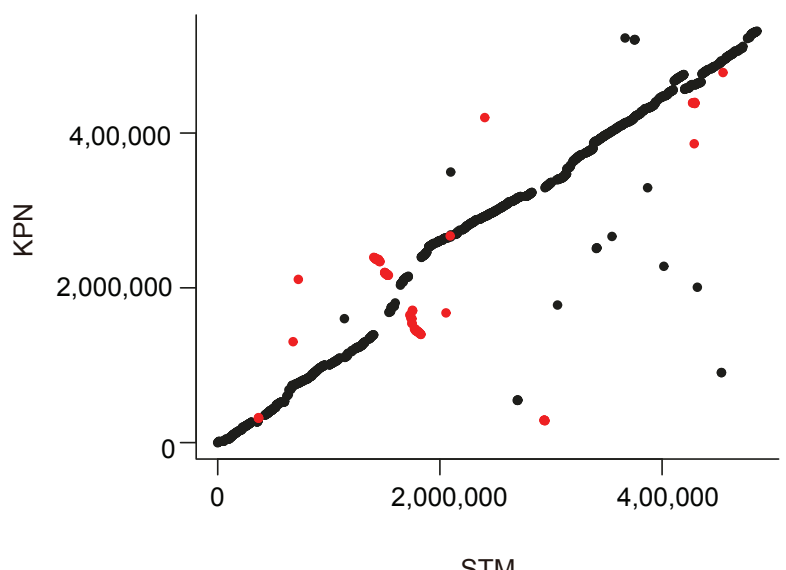

Figure 2 Dot plot for the analyzed orthologous genes in the (a) ECO-KPN; and (b) STM-KPN comparison. The $Y$ and $X$ axis represents, respectively, the chromosomal positions of the KPN genes (in base pair) and those of the orthologous genes in ECO (left panel) or STM (right panel). The black and red dots represent SOGs and COGs, respectively. Most of the COGs are located close to Ter. The positions of Ter are $1,892,000,1,549,000$ and 1,635,000 bp for KPN, ECO and STM, respectively. 
pattern. In the ECO-STM comparison, the rates of $d N$, $d S$ and $d N / d S$ of head-on-collision COGs do not differ significantly from co-orientation COGs (Additional File 8). By contrast, head-on-collision SOGs have significantly higher evolutionary rates than co-orientation SOGs. These results suggest that the higher evolutionary rates of COGs do not result from a higher proportion of head-on-collision genes. Rather, CTO by itself may be the major reason for the increased evolutionary rate.

\section{Confounding factor 5: decreased $d S$ at gene terminals (codon usage bias)}

It has been reported that the synonymous substitution rate decreases near the first 50-100 codons [12] and the last 20 codons of a gene [13], possibly because codon usage is less biased at these regions. We then ask whether SOGs tend to have lowered $d S$ at both ends as compared with COGs, so that the overall $d S$ is decreased. Accordingly, we divided each of the analyzed genes into terminal (first 50 and last 20 codons) and middle region (the rest of the codons). We then randomly sampled 70 codons from the middle region for comparison. The differences in evolutionary rates $(\Delta d S$, $\Delta d N$, and $\Delta d N / d S$ ) between the terminal codons and the randomly sampled codons were calculated and compared between COGs and SOGs. Our results show that $\Delta d S, \Delta d N$, and $\Delta d N / d S$ are similar between COGs and SOGs (Figure 3 for ECO-KPN comparison and Additional file 9 for STM-KPN comparison; all of the pairwise differences are statistically insignificant). Therefore, the difference in codon usage bias in evolutionary rate does not appear to affect our overall results.

\section{Functional enrichment analysis of COGs}

We next ask whether the rapidly evolving COGs are enriched in certain functional categories. Additional file 10 indicates that the protein products of COGs tend to be located on membranes and cell walls, and be involved in a variety of metabolic reactions. This observation is biologically sensible because (1) proteins that are located on the cell surface tend to evolve faster; and (2) different bacterial species have very different metabolic capacities. However, the relationship between CTOs and the functional preferences of these genes remains unknown.

\section{Conclusions}

To our knowledge, this is the first study to demonstrate that changes in transcriptional orientation may increase the evolutionary rates $(d N$ and $d S)$ of prokaryotic genes. We show that the evolutionary effects of CTOs are independent of gene essentiality, gene expression level, replication-transcriptional confrontation, or the decrease in $d S$ at gene terminals. However, the increase in $d N$ may be partially related to gene locations. Furthermore, our results suggest that the increases in evolutionary rates in COGs are mainly mutation-driven, as the $d N / d S$ ratios are similar between COGs and SOGs. The real cause of the increases in evolutionary rate in COGs (particularly $d S$ ) remains unclear but is worth further explorations. It is speculated that CTOs may somehow result in impediments in DNA replication (by altering, for example, DNA protein binding sites or secondary structure), which may in turn lead to the recruitment of error-prone DNA repair polymerases and the increase in mutation rate $[30,31]$.

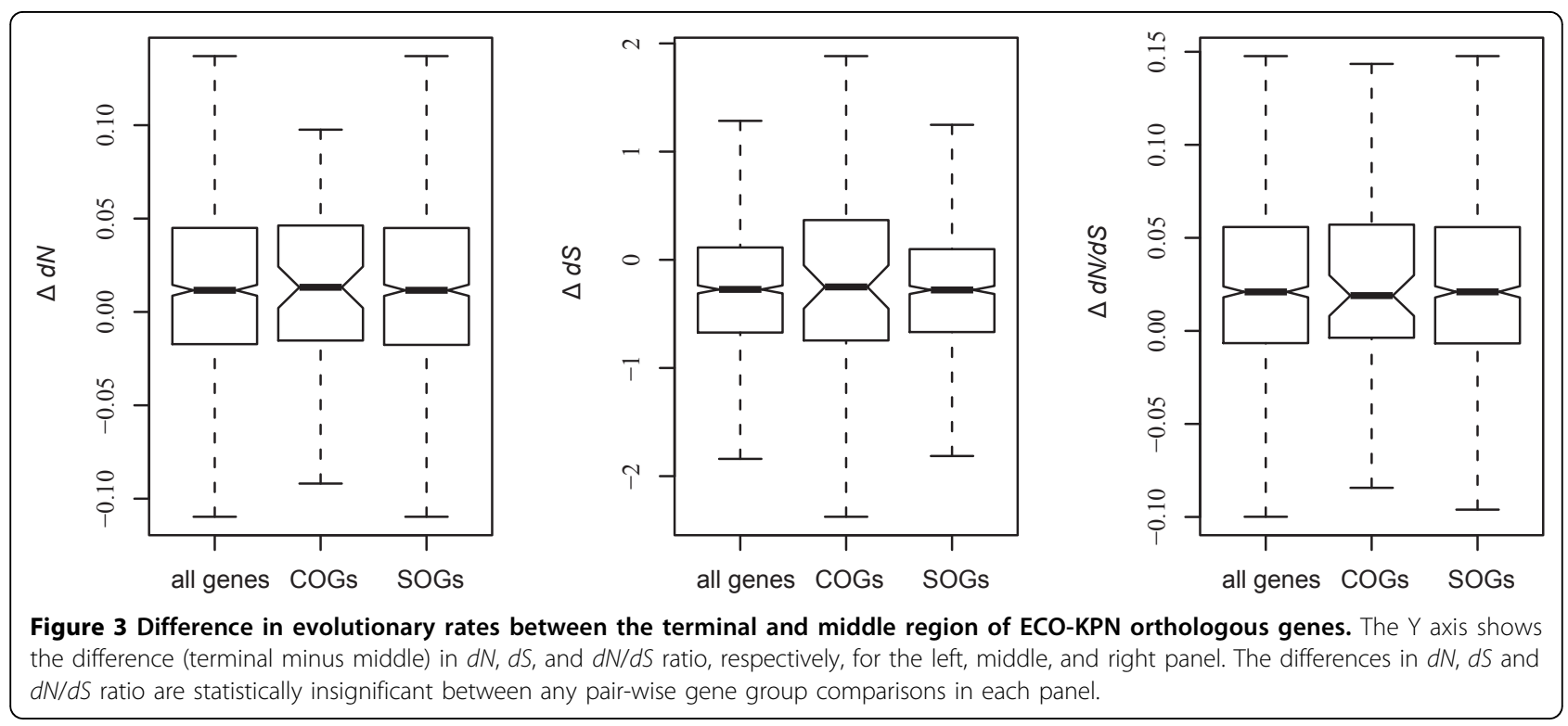




\section{Additional material}

\section{Additional file 1: The numbers and detailed information of the} orthologous gene pairs analyzed in this study.

Additional file 2: The numbers of ECO-KPN, ECO-STM, and STM-KPN orthologous gene pairs identified by reciprocal BLASTP and the OMA database

Additional file 3: The evolutionary rates of COGs and SOGs in the ECO-KPN and STM-KPN comparisons. The genes included in this analysis were identified by both reciprocal BLASTP and OMA database as orthologous genes. ${ }^{*}$ : $p$-value $<0.05{ }^{* *}$ : $p$-value $<$ $0.01{ }^{* * *}: p$-value $<0.0001$

Additional file 4: The numbers of COGs with $d S>3$ in the ECO-KPN, ECO-STM, and STM-KPN comparisons.

Additional file 5: The evolutionary rates in (a) ECO-KPN comparison; and (b) STM-KPN comparison. Here we compare the evolutionary rates of the genes that never changed transcriptional orientation in ECO, STM, and KPN, ("ESK"), the genes that changed orientation in either the ECO-STM lineage or in the KPN lineage ("ES/K"), and the genes that changed orientation in only one species ("ECO-only" or "STM-only").

Additional file 6: Comparison of the evolutionary rates of highly and non-highly expressed COGs. (a) ECO-KPN comparison at the log phase; (b) ECO-KPN comparison at the stationary phase; (c) STM-KPN comparison at the log phase; (d) STM-KPN comparison at the stationary phase. ${ }^{*}$ : $p$-value $<0.05 ;{ }^{* *}$ : $p$-value $<0.01$

Additional file 7: Partial correlations between evolutionary rates and the COG/SOG gene type while the physical distance from Ter is controlled.

Additional file 8: The evolutionary rates of COGs and SOGs that are subject to head-on collision or co-orientation between DNA replication and transcription in the ECO-STM comparison. * represents $p$ value $<0.05$.

Additional file 9: Comparison of $\Delta d N, \Delta d S$ and $\Delta d N / d S$ between COGs and SOGs in the STM-KPN comparison. $\Delta d N, \Delta d S$ and $\Delta d N / d S$ were calculated by subtracting the evolutionary rates of the middle region from those of the terminal regions of each gene. Note that none of the pair-wise comparisons in any of the three panels is statistically significant.

Additional file 10: Functional enrichment analyses for COGs in the (a) ECO-KPN and (b) ECO-STM comparisons. Note that the functional assignments were based on the gene annotations for ECO.

\section{Acknowledgements}

We thank Dr. Bernhard O. Palsson and Dr. Jay Hong for sharing the $K$. pneumoniae gene expression data. This work was supported by the intramural funding of National Health Research Institutes (NHRI), Taiwan ( $\mathrm{PH}$ 100-SP-02), and represented part of the collaborative project between NHRI and Dr. Bernhard O. Palsson's laboratory at University of California, San Diego.

This article has been published as part of BMC Bioinformatics Volume 12 Supplement 9, 2011: Proceedings of the Ninth Annual Research in Computational Molecular Biology (RECOMB) Satellite Workshop on Comparative Genomics. The full contents of the supplement are available online at http://www.biomedcentral.com/1471-2105/12?issue=S9.

\section{Author details}

${ }^{1}$ Division of Biostatistics and Bioinformatics, Institute of Population Health Sciences, National Health Research Institutes, 35 Keyen Road, Zhunan Town, Miaoli County, Taiwan, Republic of China. ${ }^{2}$ Institute of Bioinformatics and Structural Biology, National Tsing Hua University, No. 101, Section 2, KuangFu Road, Hsinchu, Taiwan, Republic of China.

\section{Authors' contributions}

$\mathrm{CHL}$ conceived the study and carried out the data analyses. $\mathrm{CHL}, \mathrm{FCC}$ and $\mathrm{CAH}$ designed the conceptual framework of the study, interpreted the results, and drafted the manuscript. CYL pre-processed gene expression data and assisted in the statistical analyses.

\section{Competing interests}

The authors declare that they have no competing interests.

Published: 5 October 2011

\section{References}

1. Mackiewicz P, Mackiewicz D, Kowalczuk M, Cebrat S: Flip-flop around the origin and terminus of replication in prokaryotic genomes. Genome Biol 2001, 2(12):INTERACTIONS1004.

2. Eisen JA, Heidelberg JF, White O, Salzberg SL: Evidence for symmetric chromosomal inversions around the replication origin in bacteria. Genome Biol 2000, 1(6):RESEARCH0011.

3. Srivatsan A, Tehranchi A, MacAlpine DM, Wang JD: Co-orientation of replication and transcription preserves genome integrity. PLOS Genet 2010, 6(1):e1000810.

4. Tillier ER, Collins RA: Replication orientation affects the rate and direction of bacterial gene evolution. J Mol Evol 2000, 51(5):459-463.

5. Blattner FR, Plunkett G 3rd, Bloch CA, Perna NT, Burland V, Riley M, ColladoVides J, Glasner JD, Rode CK, Mayhew GF, et al: The complete genome sequence of Escherichia coli K-12. Science 1997, 277(5331):1453-1462.

6. McClelland M, Sanderson KE, Spieth J, Clifton SW, Latreille P, Courtney L, Porwollik S, Ali J, Dante M, Du F, et al: Complete genome sequence of Salmonella enterica serovar Typhimurium LT2. Nature 2001, 413(6858):852-856

7. Drummond DA, Wilke CO: Mistranslation-induced protein misfolding as a dominant constraint on coding-sequence evolution. Cell 2008, 134(2):341-352.

8. Jordan IK, Rogozin IB, Wolf $\mathrm{Yl}$, Koonin EV: Essential genes are more evolutionarily conserved than are nonessential genes in bacteria. Genome Res 2002, 12(6):962-968.

9. Sharp PM, Emery LR, Zeng $K$ : Forces that influence the evolution of codon bias. Philos Trans R Soc Lond B Biol Sci 2010, 365(1544):1203-1212.

10. Mira A, Ochman $\mathrm{H}$ : Gene location and bacterial sequence divergence. Mol Biol Evol 2002, 19(8):1350-1358.

11. GenBank Database. [ftp://ftp.ncbi.nih.gov/genomes/Bacteria/]

12. Eyre-Walker A, Bulmer M: Reduced synonymous substitution rate at the start of enterobacterial genes. Nucleic Acids Res 1993, 21(19):4599-4603.

13. Eyre-Walker A: The close proximity of Escherichia coli genes: consequences for stop codon and synonymous codon use. $J \mathrm{Mol}$ Evol 1996, 42(2):73-78.

14. Altenhoff AM, Schneider A, Gonnet GH, Dessimoz C: OMA 2011: orthology inference among 1000 complete genomes. Nucleic Acids Research 2011, 39(suppl 1):D289-D294.

15. Frank AC, Lobry JR: Oriloc: prediction of replication boundaries in unannotated bacterial chromosomes. Bioinformatics 2000, 16(6):560-561.

16. Thompson JD, Higgins DG, Gibson TJ: CLUSTAL W: improving the sensitivity of progressive multiple sequence alignment through sequence weighting, position-specific gap penalties and weight matrix choice. Nucleic Acids Res 1994, 22(22):4673-4680.

17. Yang Z: PAML: a program package for phylogenetic analysis by maximum likelihood. Comput App/ Biosci 1997, 13(5):555-556.

18. Smith NG, Eyre-Walker A: Nucleotide substitution rate estimation in enterobacteria: approximate and maximum-likelihood methods lead to similar conclusions. Mol Biol Evol 2001, 18(11):2124-2126.

19. Edgar R, Domrachev M, Lash AE: Gene Expression Omnibus: NCBI gene expression and hybridization array data repository. Nucleic Acids Res 2002 30(1):207-210.

20. Huang da W, Sherman BT, Lempicki RA: Systematic and integrative analysis of large gene lists using DAVID bioinformatics resources. Nat Protoc 2009, 4(1):44-57.

21. Huang da W, Sherman BT, Lempicki RA: Bioinformatics enrichment tools: paths toward the comprehensive functional analysis of large gene lists. Nucleic Acids Res 2009, 37(1):1-13. 
22. Baba T, Ara T, Hasegawa M, Takai Y, Okumura Y, Baba M, Datsenko KA, Tomita M, Wanner BL, Mori H: Construction of Escherichia coli K-12 inframe, single-gene knockout mutants: the Keio collection. Mol Syst Biol 2006, 2:2006 0008 .

23. Drummond DA, Bloom JD, Adami C, Wilke CO, Arnold FH: Why highly expressed proteins evolve slowly. Proc Natl Acad Sci U S A 2005, 102(40):14338-14343.

24. Cherry JL: Expression level, evolutionary rate, and the cost of expression. Genome Biol Evol 2010, 2:757-769.

25. Akashi H: Gene expression and molecular evolution. Curr Opin Genet Dev 2001, 11(6):660-666.

26. Hudson RE, Bergthorsson U, Ochman H: Transcription increases multiple spontaneous point mutations in Salmonella enterica. Nucleic Acids Res 2003, 31(15):4517-4522.

27. Aguilera $\mathrm{A}$ : The connection between transcription and genomic instability. EMBO J 2002, 21(3):195-201.

28. Sharp PM, Shields DC, Wolfe KH, Li WH: Chromosomal location and evolutionary rate variation in enterobacterial genes. Science 1989, 246(4931):808-810.

29. Wang JD, Berkmen MB, Grossman AD: Genome-wide coorientation of replication and transcription reduces adverse effects on replication in Bacillus subtilis. Proc Natl Acad Sci U S A 2007, 104(13):5608-5613.

30. McDonald MJ, Wang WC, Huang HD, Leu JY: Clusters of nucleotide substitutions and insertion/deletion mutations are associated with repeat sequences. PLOS Biol 2011, 9(6):e1000622.

31. Mirkin EV, Mirkin SM: Replication Fork Stalling at Natural Impediments. Microbiol Mol Biol Rev 2007, 71(1):13-35.

doi:10.1186/1471-2105-12-S9-S19

Cite this article as: Lin et al:: Changes in transcriptional orientation are associated with increases in evolutionary rates of enterobacterial genes. BMC Bioinformatics 2011 12(Suppl 9):S19.

\section{Submit your next manuscript to BioMed Central and take full advantage of:}

- Convenient online submission

- Thorough peer review

- No space constraints or color figure charges

- Immediate publication on acceptance

- Inclusion in PubMed, CAS, Scopus and Google Scholar

- Research which is freely available for redistribution

Submit your manuscript at www.biomedcentral.com/submit 